\title{
JACKSON-STECHKIN TYPE INEQUALITY IN WEIGHTED LORENTZ SPACES
}

\author{
RAMAZAN AKGÜN AND YUNUS EMRE YILDIRIR
}

\begin{abstract}
In the present work we consider the modulus of smoothness, defined by means of the Steklov operator in weighted Lorentz spaces and prove the Jackson-Stechkin type direct theorem of trigonometric approximation. In the particular case we obtain a result on the constructive characterization of the generalized Lipschitz classes defined in these spaces. Simultaneous approximation of functions is also considered.
\end{abstract}

Mathematics subject classification (2010): 41A25, 41A27, 42A10.

Keywords and phrases: Modulus of smoothness, trigonometric polynomials, weighted Lorentz spaces, Muckenhoupt weight, direct and inverse theorem.

\section{REFERENCES}

[1] R. AKGUN, Trigonometric approximation of functions in generalized Lebesgue spaces with variable exponent, Ukrainian Math. J., 63 (2011), no. 1, pp. 1-26.

[2] H. M. Chang, R. A. Hunt And D. S. KURTZ, The Hardy-Littlewood maximal functions on $L(p, q)$ spaces with weights, Indiana Univ. Math. J. 31 (1982), 109-120.

[3] R. A. Devore, G. G. Lorentz, Constructive Approximation, Springer-Verlag (1993).

[4] Z. DitZIAN, V. TотіK, K-functionals and best polynomial approximation in weighted $L^{p}(R)$, J. Approx. Theory, (1986), no. 1, 38-41.

[5] Z. Ditzian, V. Totik, $K$-functionals and weighted moduli of smoothness, J. Approx. Theory, 63 (1990), no. 1, 3-29.

[6] Z. Ditzian, V. Totik, Moduli of Smoothness, Springer-Verlag, 1987.

[7] E. A. GADJIEva, Investigation the Properties of Functions with Quasimonotone Fourier Coefficients in Generalized Nikolskii-Besov Spaces, (Russian), Authors Summary of Candidates Dissertation, Tbilisi (1986).

[8] D. JACKSON, Uber die Genauigkeit der Annaherung stetiger Funktionen durch ganzer rationaler Funktionen gegebenen Grades und trigonometrische Summen gegebener Ordnung, Dissertation, Gottingen, 1911.

[9] V. KoKILAShVIli AND M. Krbec, Weighted inequalities in Lorentz and Orlicz spaces, World Scientific Publishing Co. Inc. River Edge, NJ, 1991.

[10] V. M. KoKILAShVILI, Y. E. YILDIRIR, On the approximation by trigonometric polynomials in weighted Lorentz spaces, J. Funct. Spaces Appl., 8 (2010), No. 1, 67-86.

[11] V. M. KoKILAshVili, Y. E. YILDIRIR, On the approximation in weighted Lebesgue spaces, Proc. A. Razmadze Math. Inst., 143 (2007), 103-113.

[12] H. N. MHASKAR, Introduction to the Theory of Weighted Polynomial Approximation, Series in Approximation and Decompisitions 7, World Scientific Publishing Co., Inc., River Edge, NJ 1996.

[13] B. Muckenhoupt, Weighted Norm Inequalities for the Hardy Maximal Function, Trans. Amer. Math. Soc. 165 (1972), 207-226.

[14] I. I. SHARAPUDINOv, Approximation of functions in $L_{2 \pi}^{p(x)}$ by trigonometric polynomials, Izv. RAN. Ser. Mat., 77 (2013), no. 2, 197-224.

[15] S. B. STECKIn, On the order of the best approximation of continuous functions, Izv. Akad. Nauk SSSR Ser. Mat. 15 (1951), 219-242. (Russian) MR 13, 29. 
[16] A. I. Stepanets, Classification and Approximation of Periodic Functions, English translation 1995, Kluwer Academic Publishers, Russian original published in Kiev by Naukova Dumka 1987.

[17] A. F. Timan, Theory of Approximaton of Functions of a Real Variable, English translation 1963, Pergamon Press, The MacMillan Co., Russian original published in Moscow by Fizmatgiz 1960.

[18] Y. E. YILDIRIR, D. M. ISRAFILOV, Approximation Theorems in weighted Lorentz spaces, Carpathian J. Math., 26 (2010), No. 1, 108-119.

[19] Y. E. YILDIRIR, D. M. ISRAFILOV, Simultaneous and converse approximation theorems in weighted Lebesgue spaces, Math. Ineq. \& Appl., 14, 2, (2011), 359-371. 